\title{
Magnetically-Dominated Accretion Flows (MDAFs) and Jet Production in the LowHard State
}

\author{
David L. Meier \\ Jet Propulsion Laboratory, California Institute of Technology
}

October 22, 2004

\begin{abstract}
In this paper I propose that the inner part of a black hole accretion inflow $\left(<100 r_{g}\right)$ may enter a magnetically-dominated, magnetosphere-like phase in which the strong, wellordered fields play a more important role than weak, turbulent fields. In the low/hard state this flow is interior to the standard ADAF usually invoked to explain the observed hot, optically thin emission. Preliminary solutions for these new MDAFs are presented.

Time-dependent X-ray and radio observations give considerable insight into these processes, and a new interpretation of the X-ray power spectrum (as arising from many disk radii) may be in order. While an evaporative ADAF model explains the noise power above $0.01 \mathrm{~Hz}$, an inner MDAF is needed to explain the high frequency cutoff near $1 \mathrm{~Hz}$, the presence of a QPO, and the production of a jet. The MDAF scenario also is consistent with the data-based, phemonenological models presented at this meeting by several authors.
\end{abstract}

Keywords: black holes, accretion, magnetic fields, jets

\section{Introduction: The 'Black Hole Problem'}

A generic, robust electrodynamic model for producing most astrophysical jets is now well understood. It has two basic requirements:

- A strong magnetic field $\left(V_{A} \equiv B /(4 \pi \rho)^{1 / 2}>>C_{S} \equiv(\Gamma p / \rho)^{1 / 2}\right.$, where $V_{A}$ is the Alfven speed and $C_{S}$ is the sound speed) that rotates fairly rapidly ( $\Omega \lesssim \Omega_{K}$, where $\Omega_{K}$ is the Keplerian angular velocity).

- Some means of loading this rotating magnetic field with plasma at fairly high elevations ( $Z \sim R$ in a cylindrical $[R, Z, \theta]$ coordinate system). Early models accomplished this loading by centrifugal action from a thin disk, but more recent studies suggest that the primary means is thermal, (heating the plasma to roughly the virial temperature; Meier, 2001).

The means by which this configuration produces a jet was first proposed by Blandford (1976) and Lovelace (1976) and has been reviewed recently by the author (Meier et al., 2001; Meier, 2004). The rotation of the magnetic field lines is retarded by the inertia of the plasma load, creating a rotating helical field configuration. Lorentz forces simultaneously push plasma up and out of the system along the rotation axis and collimate the flow with magnetic hoop stress by squeezing it toward the rotation axis, converting rotational energy of the central engine into directed kinetic outflow energy along the rotation axis.

(C) 2018 Kluwer Academic Publishers. Printed in the Netherlands. 
The jet is accelerated to the local Alfven speed by the rotational Alfven wave and beyond that to the local magnetosonic speed by the toroidal magnetic pressure gradient. The terminal velocity of the jet is approximately equal to the escape speed at the footpoint of the magnetic field in the rotating central engine.

\subsection{SPeCific Models fOR Stellar Jets}

While this model gives a general description of how astrophysical jets form, it does not answer the question of how each source produces the above two main ingredients - the global rotating magnetic field and the plasma loading. For stellar sources the first requirement is straightforward: the global magnetic field is that produced by the star's magnetosphere itself. The discovery of strong magnetic fields in pulsars and protostar systems is considered confirmation of the MHD jet production model.

The plasma loading is more problematical. In pulsars it has been shown that pair production can occur in 'spark gaps' in the very strong magnetic field $\left(10^{11-13} \mathrm{G}\right)$, producing the needed plasma inside the magnetosphere itself (Goldreich \& Julian, 1969; Ruderman \& Sutherland, 1975). In protostar systems, however, the field is not nearly as strong, so pair production cannot operate. Instead, the protoplanetary accretion disk is used to pinch the field near the equator, creating an "X-point" (Shu et al, 1994), where plasma is allosed to flow freely from the disk onto the rotating stellar field.

Finally, for jets produced in collapsing supernova cores (Wheeler et al, 2002), the plasma comes pre-loaded, since the magnetic field of the collapsing core has become threaded into the progenitor mantle during the late stages of stellar evolution. It is the rapid rotation of the core that is suddenly and rapidly generated, by the collapse of that iron core to proto-neutron star densities.

\subsection{The Black Hole Problem}

While it is clear that black hole systems often produce fast and powerful jets, they present a serious challenge to the electromagnetic theory of jet production. The plasma loading itself is a relatively easy problem to solve. The fact that black hole systems that produce jets are associated with hot accretion flows (Fender et al., 1999) indicates that the plasma must be loaded onto the field lines from the accretion flow by some thermal means. 
However, by themselves, black holes cannot support a magnetic field ${ }^{1}$. They can have such a field only if there is an external supply of plasma in which currents generate magnetic flux that can then thread the black hole. In the absence of a significant amount of external plasma, a black hole loses its field in a few light-crossing times (Thorne, Price, \& MacDonald, 1986). The field, therefore, must be supported by currents in the black hole accretion flow. This conclusion itself presents a problem, however. Accretion disks are believed to be weakly-magnetized plasmas in highly turbulent, orbital flow about the black hole (Balbus \& Hawley, 1998). How does a global, wellordered rotating magnetosphere develop naturally from a turbulent accretion disk?

In addition, even if a global magnetosphere can be constructed, there is a question as to how that magnetosphere can couple to black hole rotation to produce a strong jet. While the accretion disk itself can produce rotation of the magnetic field, it cannot be the main source of jet power in many jet-producing supermassive black hole systems. Radio galaxies and quasars that have similar optical properties, and therefore similar accretion disks, can differ in their radio jet luminosities by factors of $10^{5-6}$. This is most easily explained by tying jet production to rotation of the central object, just as it is done in stellar jet-producing systems. In addition, black hole systems are known to produce jets $\sim 30$ times stronger than those from neutron stars with similar accretion rates (Migliari et al., 2003). While this comparison may be complicated by effects of the neutron stars' magnetic field, a strong coupling of jet production to the black hole spin also may be at work.

To solve this problem we will assume here that the "magnetic Penrose" mechanism of extracting rotational energy is at work (Koide et al., 2002): if plasma threaded with a magnetic field enters the ergosphere, then that plasma can be accelerated in a direction opposite to the black hole's spin, acquiring negative energy and angular momentum in the process. Positive energy and angular momentum then is transferred to the rotating magnetic field, which uses that to accelerate and collimate the jet.

The purpose of this paper is to explore answers to the two remaining questions: 1) how does a turbulent, magnetized disk create a global, wellordered magnetic field that can couple to the black hole rotation and 2) how does the accretion disk load the field lines with plasma?

\footnotetext{
1 It is true that a charged, rotating black hole has a dipole magnetic moment (Misner, Thorne, \& Wheeler, 1973). However, a strongly charged black hole will induce charge separation in any surrounding plasma, accreting charges opposite in sign to that on the hole and expelling those of like sign. This will effectively discharge the hole in a few light-crossing times.
} 


\section{Basics of Magnetically-Dominated Accretion Flows}

\subsection{WHAT IS AN MDAF?}

An MDAF is an accretion flow in which the magnetic forces dominate over the thermal and radiation forces. In a normal accretion disk model, the weak magnetic field creates a "magneto-rotational instability" (MRI) (Balbus \& Hawley, 1998) in which turbulence dominates the angular momentum transport and the eddy turnover time $\tau_{\text {turb }}$ is shorter than the inflow time $\tau_{\text {inflow }} \equiv$ $R / V_{R}$. A steady disk structure develops in which magnetic field components $B_{R} \sim B_{\phi} \propto R^{-5 / 4}$ and pressure scales as $p \propto R^{-3 / 2}$. The ratio of magnetic to thermal forces $\alpha \propto B_{R} B_{\phi} / p$ remains constant at $\sim 0.01-1.0$.

We recognize two types of magnetically-dominated accretion flows. The first is still turbulent, but now the ratio of the time scales is reversed: $\tau_{\text {inflow }}<$ $\tau_{t u r b}$. Small eddies continue to transport angular momentum, but the larger ones are stretched out in the $R$ direction before they have a chance to turn over. In this case, $B_{R} \propto R^{-5 / 2}$ and $B_{\phi} \propto R^{-1 / 2}$ decouple and $p \propto R^{-3 / 2}$, so that magnetic stresses increase as $R$ decreases: $\alpha \propto R^{-3 / 2}$. We call this type of flow "transitional", because it connects a turbulent flow with $\alpha<1$ to one with $\alpha>1$ and the MRI turned off. If $\alpha_{0}$ is the value at $R_{0}$, and $R_{1}$ is the radius where $\alpha$ attains unit value, then

$$
R_{1} / R_{0}=\alpha_{0}^{-2 / 3}
$$

If $\alpha_{0} \sim 0.3$, as is expected in advection-dominated accretion flows (Narayan et al., 1998), then $R_{1} / R_{0} \sim 0.5$. So, if the interior of an ADAF becomes magnetically dominated, the transition region will be rather narrow in radius.

In the second type of MDAF, which is a solution to "Gammie flow" (Gammie, 1999), MRI turbulence has ceased and the inflow is laminar along strong magnetic field lines. $B_{R} \propto R^{-3 / 2}$ and $B_{\phi} \propto R^{-1}$ are still decoupled. The thermal pressure scaling depends critically on the energy balance in the gas now, but simple models indicate $p \propto R^{-1 / 2}$. So $\alpha \propto R^{-2}$ continues to increase inward, and the flow continues to become more magnetically dominated as it approaches the black hole. Figure 1 shows a schematic of our low-state model and will be discussed more fully below.

MDAF-type solutions are seen in MRI simulations only in the plunging region very near the black hole, not out to distances as large as $\sim 100 r_{g}$. Why? The reason may be the assumption implicit in the simulations that the flow is radiatively inefficient. This may be the case for $R \gtrsim 100 r_{g}$ (where $r_{g} \equiv G M / c^{2}$ ); in this case the temperature remains $T \lesssim 5 \times 10^{9} \mathrm{~K}$. However, inside this radius electrons radiate copiously by synchrotron, pair production, and other relativistic processes. While ADAF models assume that the ion temperature can remain hot $\left(T_{i} \sim 10^{12} \mathrm{~K}\left(R / r_{g}\right)^{-1}\right)$, if there is strong coupling between ions and electrons, the ions will cool to the $\gtrsim 10^{9} \mathrm{~K}$ temperature as 


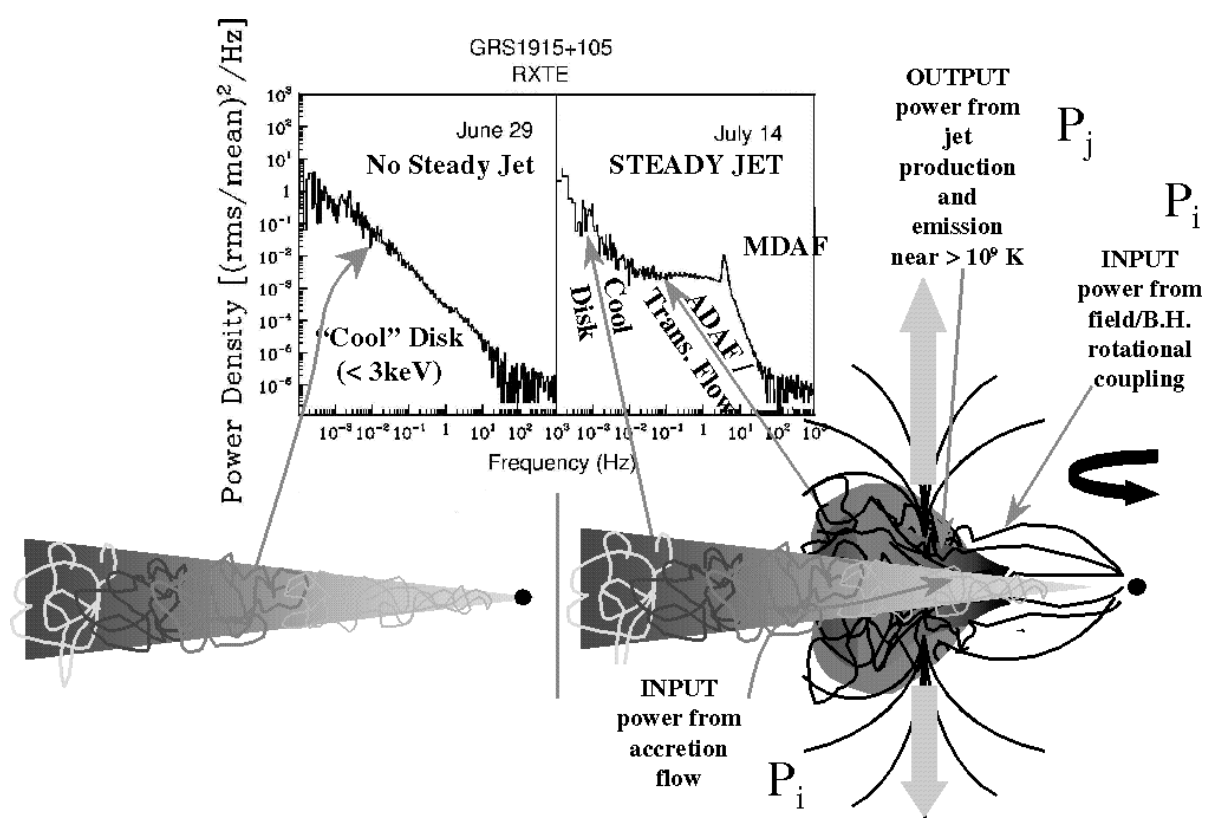

Figure 1. Schematic diagram of the MDAF model for GRS 1915+105. Power spectrum data are taken from Morgan et al. (1997). A cool disk fits the power spectrum in the high state (left) when no jet is produced. In the hard state (right) we not only need an ADAF (extended corona) but also an MDAF (inward-facing magnetosphere) to produce the few $\mathrm{Hz}$ cutoff and QPO, and an outward-facing magnetosphere to produce the jet. The jet will be launched from the transition radius near $\sim 100 r_{g}$. The input and output powers $\mathrm{P}_{i, j}$ correspond to those in the Malzac et al. model. An MDAF is also expected in some intermediate accretion states when the cool disk is still truncated at a radius $>r_{g}$.

well. It is often assumed that such cooling would lead once again to a geometrically thin, optically thick disk. However, these models show that there is another solution: cool, but still optically thin flow along strong magnetic field lines. It, therefore, is extremely important to begin performing MRI simulations with a real energy equation, including separate evolution of the ions and electrons.

\subsection{What ARE THE Properties OF MDAFs?}

The inner MDAF is an extraordinarily inefficient flow. It is a nearly-radial in-spiral, geometrically thick because of magnetic pressure support (Meier, 2004). Virtually all orbital angular momentum is transferred out to $R_{1}$ along the strong field lines. The plasma experiences only compressional heating and radiative cooling by electrons and could remain quite cool; the majority of the 
gravitational energy released is converted into radial infall kinetic energy, not heat.

Because the magnetic radial channels are potentially distinct, the flow may break up into inhomogeneous "spokes". A signature of an MDAF may be a quasi-periodic oscillation at one or more of two transition radius frequencies: 1) the orbital/Alfven frequency

$$
\mathrm{v}_{A}=V_{A} / 2 \pi R_{1}=1.1 \mathrm{~Hz}\left(M_{\bullet} / 10 M_{\odot}\right)^{-1}
$$

and 2) the MHD slow mode ("organ pipe") frequency

$$
\mathrm{v}_{S}=V_{S} / 2 \pi R_{1} \approx C_{S} / 2 \pi R_{1}=0.02-0.13 \mathrm{v}_{A}
$$

or $\sim 20-140 \mathrm{mHz}$ for a $10 M_{\odot}$ black hole, depending on the azimuthal length of the resonating magnetic tubes. Because $v_{S}$ is excited acoustically near $R_{1}$ along the length of these tubes, it may be characterized by multiple harmonics, whereas the orbital/Alfven mode should be rather pure.

The magnetic field lines extending inward toward the black hole may tap the hole's rotational energy if they penetrate the ergosphere. However, in general the hole rotation rate will not match the, usually slower, $v_{A}$. One therefore might expect an episodic interaction, where the field enters the ergosphere, is wound up rapidly, reconnects in a series of rapid flares separated by the ergosphere rotation time, and finally pulls back from the hole for a secular time. The behavior of SgrA at the Galactic center (Genzel et al., 2003) is very similar to what might be expected from a rotating black hole/MDAF interaction.

In addition to an inner magnetosphere of closed field lines reaching toward the black hole, there also may be open field lines extending from $R_{1}$ to infinity (see Fig. 1 and Meier, 2004). The transition radius, therefore, has all the properties necessary to launch a jet: the base of large-scale, open, rotating magnetic field lines being loaded with hot ADAF material. Excess angular momentum is deposited at $R_{1}$ by the radial field lines that connect periodically

to the black hole ergosphere. An outflowing MHD wind/jet would be a good candidate for carrying off this excess angular momentum.

\section{Discussion}

\subsection{MDAFs and the Low/Hard (Plateau) State of XRBs}

When the transient X-ray binary (XRB) source GRS $1915+105$ is in the soft state and not producing a jet, its photon spectrum is dominated by a cool thermal spectrum, and its power spectrum is a rather featureless power law of $d P / d v \propto v^{-4 / 3}$. When the source begins to produce a steady jet, it enters 
a low/hard state in which the photon emission is dominated by a non-thermal spectrum. And the power spectrum develops bandwidth-limited noise (a flat $\left(d P / d v \propto v^{0}\right.$ shoulder with a steep cutoff above $\left.\sim 3 \mathrm{~Hz}\right)$ and a QPO at $1-3$ $\mathrm{Hz}$. It is natural to associate the non-thermal photon spectrum and bandwidthlimited noise with the optically thin, turbulent ADAF that has formed in the center of the disk. But what produces the QPO, and why would the ADAF be bandwidth-limited? Why does its turbulence not extend all the way to the natural frequencies near the black hole $(\sim 100 \mathrm{~Hz})$ ?

The inner MDAF model provides natural answers to these questions. While the thin accretion disk is truncated by the ADAF at, perhaps, $\sim 1000 r_{g}$, the ADAF itself is truncated at $R_{1} \sim 100 r_{g}$ by the MDAF, cutting off the ADAF turbulence above a few $\mathrm{Hz}$. The slope of this cutoff may represent the high frequency tail of the turbulence spectrum near $R_{1}$. The QPO is produced by the magnetic flux tubes that stretch toward the black hole and rotate at roughly the orbital $R_{1}$ frequency — again a few $\mathrm{Hz}$.

The power spectrum at each disk radius should be dominated by a rather narrowly-peaked local spectrum (Maron \& Blackman, 2002). We therefore can approximate the total disk power spectrum by assuming the local spectrum to be a delta function and simply plotting the variation of the turbulence strength with radius against the variation of the principal local (orbital) frequency with radius:

$$
P[r(v)]=(2 \pi \Delta R \delta H) \rho V_{t u r b}(R)^{2}
$$

where $\Delta R \sim R$ is the annulus over which $\rho$ and $V_{\text {turb }}$ remain roughly constant, and $\delta H$ is the skin depth over which the turbulent eddies can be seen by the observer (roughly the optical depth). For an $\alpha$-disk (Shakura \& Sunyaev, 1973), we find $P(r) \propto R^{1 / 2} \propto v^{-1 / 3}$, or $d P / d v \propto v^{-4 / 3}$, in agreement with GRS $1915+105$ in the high state. However, in the low state, for a simple evaporative ADAF model $\left(\dot{M} \propto R^{-1}\right)$ (Esin et al., 1997), we find that $d P / d \vee \propto$ $v^{-2 / 3}$, which is not flat. In order to obtain $d P / d v \propto v^{0}$, we need to assume a steeper rate of evaporation of the thin disk into the ADAF: $\dot{M} \propto R^{-2}$. Detailed modeling of the power spectrum as disk turbulence at different radii may, therefore, become an important diagnostic of conditions in the optically thin portion of the accretion flow, ADAF and MDAF alike.

Figure 1 shows a schematic picture of GRS 1915+105 in the soft and hard states, the corresponding power spectra, and energy inputs to and outputs from the transition region.

\subsection{Relation to Presentations on XRBs at this Meeting}

Fender et al. (2004) have proposed a phenomenological model for jet production in which the jet speed increases as the inner disk radius decreases. This model explains why strong jet outbursts are seen when the disk transitions 
to the high/soft state and not when it transitions to the low/hard state: the jet speed decreases with time in the latter case, resulting in no formation of a shock.

This model fits well with the MDAF scenario. We identify the low/hard state as one in which the cool accretion disk completely evaporates before the ADAF transitions into an MDAF, i.e. for $R>R_{1}$. In this case, near the central engine, the jet speed is simply the escape speed from the transition radius, or

$$
V_{\text {jet }} \sim 2 G M / R_{1}
$$

which gives a non-relativistic jet of $V_{\text {jet }} \sim 0.1 \mathrm{c}$. Eventually, as the accretion rate is increased, the cool disk begins to extend inside $\sim 100 r_{g}$, and the ADAF changes from an accretion flow in its own right to simply a corona above a dense cool disk. The ADAF no longer extends inside the truncated cool disk; that region is filled with the MDAF only, extending from the ADAF corona inward. This begins the move toward the high state along the upper horizontal branch in the intensity/hardness plane: the hard ADAF emission begins to be suppressed, the thermal emission from the cool disk gains in strength, the radius $R_{1}$ where the transition to MDAF occurs now follows the cool disk truncation radius. The jet velocity from equation (5) increases as the disk truncation radius decreases. Eventually $R_{1}$ reaches all the way to the black hole horizon, and the MDAF is swallowed. This turns off the jet, but not before its velocity reaches close to $c$ as $R_{1} \rightarrow r_{H}$, the horizon radius. It is this fast jet that creates the shock and outburst that we observe.

Malzac et al. (2004) also have interpreted the variability of XTE J1118+480 as coupling between the corona and the jet through a common reservoir where large amounts of accretion power are stored. In the MDAF model we identify the transition region at $R_{1}$ as this reservoir. Energy and angular momentum input into this region comes from two sources: the accretion flow from outside and the magnetic coupling to the black hole from inside $R_{1}$. The output power is the jet production that occurs at this radius. It is important to note that the predicted temperature at this transition region is of order a few $\times 10^{9} \mathrm{~K}$, and it lies at $\sim 100 r_{g}$ in the low state, but can move inward as the accretion rate increases (see above).

\subsection{MDAFS AND LOW-LUMINOSITY AGN}

Black hole accretion in active galactic nuclei (AGN) is expected to act similarly to that in XRB systems: bright Seyfert and quasar objects are believed to be in a soft state while those AGN without strong optical line emission (low-luminosity AGN [LLAGN], FR I radio galaxies, Sgr A) are believed to be in a low/hard state. While there is some timing data available on these latter objectes, a detailed comparison with the MDAF model is not possible 
at this time as a QPO-producing plateau state has not yet been identified. Our discussion of MDAFs in AGN therefore will be more speculative.

LLAGN do indeed show bandwidth-limited noise, and the cutoff/break at high frequency sometimes is used as an indicator of black hole mass, with $\tau_{b r} \approx 7.7 \mathrm{~d}\left(M_{\bullet} / 10^{7} M_{\odot}\right)$, where $\tau_{b r}$ is the time scale, in days, where the break occurs in the AGN X-ray power fluctuation spectrum Papadakis (2004). The MDAF model provides a physical reason why this ad hoc scaling of the break in different systems is a reasonable black hole mass indicator. In the model the frequency of this break will be equal to, or slightly greater than, $v_{A}$ (equation 2), so $\tau_{b r}=1 / v_{A} \approx 10 \mathrm{~d}\left(M_{\bullet} / 10^{7} M_{\odot}\right)$.

AGN also display another property similar to that shown by X-ray binaries, and the MDAF model provides the same explanation there as well. Jets produced by quasars and many Seyferts tend to be quite relativistic, even within only a parsec from the black hole core. They therefore may be launched and accelerated rather close to the central black hole. This suggestion is supported by semi-analytic jet acceleration models, which suggest a magnetic foot point only a few gravitational radii from the hole for 3C 345 (Vlahakis \& Konigl, 2004). However, jets produced by LLAGN and FR Is (and their counterparts, the BL Lacertae objects) are either less relativistic or show no motion at all. A similar model for acceleration of the NGC 6251 jet yields an inner foot-point of $\sim 34 r_{g}$ for a $6 \times 10^{8} M_{\odot}$ black hole (Vlahakis \& Konigl, 2004). Furthermore, M87 shows significant collimation on scales of $60-200 r_{g}$ (Biretta et al., 2002), and its jet speed at a distance of $0.16 \mathrm{pc}$ from the core is only $0.1 \mathrm{c}$. Yet, at kiloparsec distances, M87 shows superluminal motions up to $6 \mathrm{c}$. Jets in AGN systems identified with the low/hard state appear to be launched with smaller velocities and at larger distances from the central black hole.

It appears possible, then, that the jet-production region in LLAGN and FR I objects also may look like that in Figure 1, with the launch point lying many tens of gravitational radii from the black hole. Only through continual, and persistent acceleration by the black hole over large vertical distances (many parsecs to kiloparsecs) do jets in low/hard state AGN achieve the relativistic speeds observed very far downstream of the accretion disk.

\section{Conclusions}

Interpretation of the photon and power spectra of black hole systems like GRS 1915+105 leads to a new magnetically-dominated accretion flow (MDAF) model for the low/hard state with three distinct disk regions:

1. As in previous models, the outer region of the disk is a geometrically thin, optically thick, and cool turbulent disk, driven by the MRI. 
2. Likewise, at intermediate radii $\left(\sim 100-1000 r_{g}\right)$ there is a one-temperature, advection-dominated, turbulent accretion flow (ADAF) disk/corona that is geometrically thick, optically thin, and hot. An evaporation rate into this corona that scales as $\dot{M} \propto R^{-2}$ is more consistent with the power spectrum than other models.

3. The structure inside $\sim 100 r_{g}$ distinguishes this model from others: at the radius where cooling by relativistic electrons becomes important, the ADAF transitions to an MDAF with $\alpha>>1$. The inflow is extremely inefficient, non-turbulent and nearly radial along strong magnetic field lines - essentially an inward-facing magnetosphere. The narrow annulus where the flow transitions from ADAF to MDAF is an ideal site for open field lines and the launching an MHD-powered jet.

We identify the bandwidth-limited noise that appears in the low/hard state as the ADAF's MRI turbulence viewed through the optically thin flow. The MDAF model predicts the observed truncation of that noise at a few $\mathrm{Hz}$ and the appearance of a strong QPO at the same place, as well as the very low frequency QPOs at $0.01-0.1 \mathrm{~Hz}$. Finally, the MDAF model is consistent with the pheomenological models of Fender et al. (2004) and Malzac et al. (2004) and provides a physical connection between them and black hole accretion theory. In particular, extension of this model to include an MDAF inside all truncated disks naturally predicts the variation in jet speed with inner radius deduced by Fender et al. (2004).

The model suggests a new interpretation of the power spectrum of black hole candidates: like the photon spectrum, each small range in frequency $\Delta v$ is contributed by a given annulus $\Delta R$ in the accretion disk, with the central frequency corresponding to the Keplerian frequency at that radius. The spectral slopes are due not to the physics of the turbulence itself but rather to variations in disk structure with radius. Only the cutoff at a few $\mathrm{Hz}$ is indicative of the (high frequency end of the) local power spectrum there.

\section{Acknowledgements}

The author is especially grateful to T. Macarone and R. Fender for organizing this conference on black hole accretion on all mass scales. Emphasizing the similarities and scaling of black hole systems contributes greatly to their overall understanding. The author is supported, in part, by a NASA Astrophysics Theory Program grant. This research was performed at the Jet Propulsion Laboratory, California Institute of Technology, under contract to NASA. 


\section{References}

Balbus, S.A. \& Hawley, J.F. Rev. Mod. Phys., 70:1-53, 1998.

Biretta, J.A., et al. Ap. J., 520:621-626, 1999.

Biretta, J.A., et al. New Astr. Rev., 46:239-45, 2002.

Blandford, R. D. M.N.R.A.S., 176:465, 1976.

Esin, A.A. et al. Ap. J., 489:865, 1997.

Fender, R.P. et al. Ap. J., 519:L165, 1999.

Fender, R.P. et al. M.N.R.A.S., 355:1105-1118, 2004.

Gammie, C.F. Ap. J., 522:L57-L60, 1999.

Genzel, R. et al. Nature, 425:934-937, 2003.

Goldreich, P. \& Julian, W. H. Ap. J., 157:869-880, 1969.

Koide, S., et al. Science, 295:1688, 2002.

Lovelace, R. Nature, 262:649, 1976.

Malzac, J. et al. M.N.R.A.S., 351:253, 2004.

Maron, J. \& Blackman, E.G. Ap. J., 566:L41-L44, 2002.

Meier, D. L. Ap. J., 548:L9-L12, 2001.

Meier, D. L. Proc. of the X-ray Timing Meeting: Rossi \& Beyond, eds. P. Kaaret \& J. Swank, in press, 2004.

Meier, D. L., Koide, S., \& Uchida, Y. Science, 291:84-92.

Migliari, S. et al. M.N.R.A.S., 342:L67, 2003.

Misner, C., Thorne, K.S., \& Wheeler, J.A. Gravitation, Freeman, 1973.

Morgan, E.H. et al.. Ap. J., 482:993-1010, 1997.

Narayan, R., Mahadevan, R., \& Quataert, E. The Theory of Black Hole Accretion Disks, eds. Abramowicz, Bjornsson, \& Pringle, Cambridge Univ. Press, Cambridge, 1998.

Papadakis, I.E. M.N.R.A.S., 348:207, 2004.

Ruderman, M.A. \& Sutherland, P.G. Ap. J., 196:51-72, 1975.

Shu, F. et al.. Ap. J., 429:781-796, 1994.

Shakura, N.I., \& Sunyaev, R.A. Astron. Astroph., 24:337-355, 1973.

Thorne, K.S., Price, R., \& MacDonald, D.A. Black Holes: The Membrane Paradigm, Yale Univ. Press, New Haven, 1986.

Vlahakis, N. \& Konigl, A. Ap. J., 605:656-61, 2004.

Wheeler, J.C., Meier, D.L., \& Wilson, J. Ap. J., 568:807-819, 2002. 
Meier.tex; 28/09/2018; 9:44; p.12 